Discussion Paper No. 05-11

\title{
Yardstick Competition and Policy Innovation
}

Johannes Rincke

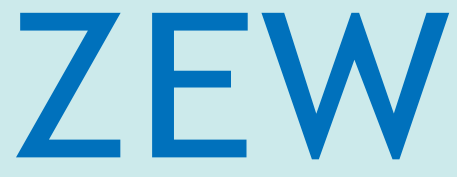

Zentrum für Europäische Wirtschaftsforschung $\mathrm{GmbH}$

Centre for European

Economic Research 
Discussion Paper No. 05-11

\title{
Yardstick Competition and Policy Innovation
}

\author{
Johannes Rincke
}

Download this ZEW Discussion Paper from our ftp server:

\section{ftp://ftp.zew.de/pub/zew-docs/dp/dp0511.pdf}

Die Discussion Papers dienen einer möglichst schnellen Verbreitung von neueren Forschungsarbeiten des ZEW. Die Beiträge liegen in alleiniger Verantwortung der Autoren und stellen nicht notwendigerweise die Meinung des ZEW dar.

Discussion Papers are intended to make results of ZEW research promptly available to other economists in order to encourage discussion and suggestions for revisions. The authors are solely responsible for the contents which do not necessarily represent the opinion of the ZEW. 


\section{Non-Technical Summary}

The political agency problem between voters and their elected representatives is one of the fundamental problems in political economics. The core of this problem can be described very simply: Political representatives are often better informed than voters about the prospects of all kind of public projects as well as the costs of providing public goods and services. Furthermore, the ability of voters to monitor their representatives is limited, too. Hence, if governments invest in wasteful projects, give favors to special interests or engage in some sort of rent-seeking from public projects, it is difficult for the voter to find out about this: limited information often makes it impossible for the voter to distinguish such behavior from benevolent and honest political activities under unfavorable circumstances. To put it shortly, the ability of voters to assess the absolute quality of public policies is, in general, rather limited. This paper focuses on the problem of policy innovations, i.e. the choice between traditional and new political technologies. Of course, the voter always wants optimal policies to be implemented, but often it is difficult to find out whether a new policy with an unknown payoff or a traditional policy with a known outcome is the best choice. At the same time, governments often prefer to run known policies because they are less demanding. Hence, governments tend to avoid policy innovations while pretending that what they do is the optimum the voter can get. The paper addresses the question whether in decentralized political systems, voters can benefit from the comparison of policies and outcomes across jurisdictions. The basic idea is that comparative performance evaluation ('yardstick competition') across jurisdictions may discipline local governments such that optimal policies are chosen more often than under centralized government. In a theoretical analysis it is shown that under certain conditions, yardstick competition indeed promotes policy innovation. The paper thus supports the view that decentralized systems of governments offer favorable conditions for policy innovation. The model also has empirical implications that will be tested in future empirical work on policy innovations in federal systems. 


\title{
Yardstick Competition and Policy Innovation
}

\author{
Johannes Rincke
}

February 2005

\begin{abstract}
A simple model of yardstick competition between jurisdictions is presented. Governments of jurisdictions face the alternative to choose between an old and a new policy with stochastic payoffs. The new policy is superior to the old policy in one state of the world, and inferior in the other. Governments are either benevolent, serving the interest of the voter, or rent-seeking. An equilibrium with yardstick competition is shown to exist where bad governments having a good government in their neighborhood choose the new policy more often compared to an equilibrium without relative performance evaluation. Overall, the probability of policy innovations is increased by yardstick competition. The model has a testable empirical implication saying that policy innovations should show spatial correlation.
\end{abstract}

${ }^{*}$ Centre for European Economic Research (ZEW) and Mannheim University

\section{Correspondence:}

Johannes Rincke

ZEW

L7, 1

D-68161 Mannheim

phone: $+49 / 621 / 1235-217$

email: rincke@zew.de 


\section{Introduction}

The political agency problem between voters and their elected representatives is one of the fundamental problems in political economics. The core of this problem is a severe informational asymmetry: Representatives are better informed than voters about the prospects of all kind of public projects as well as the costs of providing public goods and services. Furthermore, the ability of voters to monitor their representatives is limited, too. Thus investments in wasteful projects, favors to special interests and rent-seeking by politicians often cannot be distinguished from benevolent and honest political activities under unfavorable circumstances. Repeated elections are the basic means the electorate can use to sort the bad representatives from the good. But given that it is difficult for voters to assess the performance of their representatives and to distinguish between bad performance and bad luck, elections alone clearly do not work well as a discipline device for politicians. In decentralized political systems, however, voters may base their decision at the ballot box on comparative rather than absolute performance (or both). In correlated environments, inference on the quality of a jurisdiction's performance will be more precise if it is based on a performance comparison across several jurisdictions. In their strategic interaction with the electorate, representatives will anticipate the comparative performance evaluation and adjust their behavior. In recent years, the resulting political yardstick competition between jurisdictions and its effect on political accountability has been a much discussed topic in the public economics literature.

In this paper, the question of policy innovation in the presence of yardstick competition is addressed. In a simple model with two jurisdictions, governments face the alternative to choose between an old and a new policy with stochastic payoffs. The new policy is superior to the old policy in one state of the world, and inferior in the other. Choosing the new policy is costly for governments since they have to invest some additional effort to per- 
form it. Together with a policy, governments choose transfers to themselves, thereby reducing the share of policy payoffs going to the electorate. There are two types of governments, benevolent ('good') and rent-seeking ('bad'). The model has two time periods, with elections taking place at the end of the first period.

The main results of the model are the following. Without yardstick competition, bad governments prefer the old policy in all cases. This is because the new policy is costly to perform and bad governments cannot increase their reelection probability by switching from the old to the new policy. With yardstick competition, however, an equilibrium is shown to exist where the overall probability of innovations conditional on the new policy being superior is higher than without yardstick competition. In this equilibrium, bad governments seeking reelection and expecting to see innovations by good governments in their neighborhood choose the new policy. This is because successfully innovating good governments reveal that the new policy is superior. Bad governments seeking reelection therefore have to switch to the innovation, too. These findings also carry over to an extended model with a multi-dimensional policy space. In a situation where voters base their inference on the quality of policies at home on the performance of their governments relative to nearby rather than to more distant jurisdictions, the model has a testable empirical implication saying that policy innovations should show positive spatial correlation.

The paper is related to a number of papers in the growing body of literature on political agency problems. In one of the fundamental contributions in the field, Coate and Morris (1995) show how asymmetric information about policies ('policy uncertainty') and politicians ('politician uncertainty') in a world with rational voters can explain that transfers to special interests are often made inefficiently. Besley and Case (1995) introduced yardstick competition between jurisdictions as a discipline device for rent-seeking politicians. They show how the asymmetric information problem between politicians and vot- 
ers can in correlated environments be alleviated by comparative performance evaluation across jurisdictions. ${ }^{1}$ This paper extends the model of Besley and Case (1995) to allow for strategic interaction between jurisdictions with respect to policy innovations as discrete choice decisions. The analysis is also extended to a multi-dimensional policy space. Kotsogiannis and Schwager (2003) discuss the effect of outside information on the incentives to experiment with innovative public policies. They show that outside information is a two-sided edge: it is beneficial since it helps the voter to separate selfish from benevolent politicians, but it also creates an externality that reduces the incentives to engage in policy experiments. This paper takes a different approach and assumes that incumbents do not have to experiment in order to find out about optimal policies. Instead, the paper discusses the role of outside information provided by observable policy decisions in other jurisdictions in a situation where for incumbents choosing the optimal policy is sometimes costly. In a recent contribution, Kotsogiannis and Schwager (2004) discuss the effect of career concerns of politicians in federal systems on their willingness to experiment with new policies. Recent contributions that deal with the role of yardstick competition in political agency problems include Besley and Smart (2002), who discuss the effect of yardstick competition on public good provision and wasteful spending, Belleflamme and Hindriks (2002), extending the approach of Coate and Morris (1995) to a multi-agency framework, and Wrede (2001), who provides a model with rent-seeking politicians and yardstick competition in a more richly modelled political system.

The remainder of the paper is organized as follows. Section 2 presents the model. In section 3, Perfect Bayesian Equilibria for the model with a single jurisdiction and with yardstick competition between two jurisdictions are analyzed. An extension of the model with a multi-dimensional policy space is discussed in section 4 . Section 5 concludes.

\footnotetext{
${ }^{1}$ In the principal-agent theory more general incentive schemes involving yardstick competition have been analyzed. See, for example, Holmstrom (1982).
} 


\section{The model}

Consider two jurisdictions $i=1,2$. Each jurisdiction is populated by a representative resident and has a government which performs a public policy $p_{i}$ generating a payoff $\pi_{i}$. Governments face the alternative to choose between an old policy $o$ and a new policy $n$. The payoff from the old policy is either $\pi$ or $\pi+\Delta$, where $\Delta>0$. There are two states of the world, $s \in\{1,2\}$. In state 1 , the new policy either generates payoff $\pi+3 \Delta$ or $\pi+2 \Delta$ and is therefore superior to the old policy. In state 2, the new policy has payoffs $\pi-\Delta$ or $\pi-2 \Delta$. Hence, in state 2 the new policy is inferior to the old policy. Residents derive per-period utility $v_{i}=\pi_{i}-\tau_{i}$, where $\tau_{i}$ is a transfer the government can make to itself. Governments are either benevolent or rent-seeking. Good governments behave non-strategically and always choose the policy with the higher payoff together with a zero transfer in order to maximize the representative resident's utility. Bad governments' per-period utility is

$$
u_{i}\left(p_{i}, \tau_{i}\right)= \begin{cases}\tau_{i}-e & \text { if } \quad p_{i}=n \\ \tau_{i} & \text { if } \quad p_{i}=o,\end{cases}
$$

where $e>0$ is a special effort needed to perform $n .^{2}$ Let the transfer be restricted to $\tau \in\{0, \Delta, \bar{\tau}\}$ and assume $\bar{\tau}>\Delta>e$ as well as $\bar{\tau}<3 \Delta .{ }^{3}$ The former assumption means that we allow for transfers exceeding the difference between the high and the low payoff given a certain state of the world and a certain policy, and that we require that choosing the new policy and taking a transfer that equals this difference generates a positive per-period utility for incumbents. The latter assumption guarantees that, given that the new policy is used, the lowest possible per-period utility of the voter in state 1 is

${ }^{2}$ One could see $e$ as the effort needed to perform the new policy in excess of the effort needed to perform the old policy.

${ }^{3}$ The assumption that the choice of transfers is discrete is not restrictive. With $\tau \in[0, \bar{\tau}]$ instead the crucial properties of the equilibria discussed in the following are the same. 
higher than the highest per-period utility in state 2 .

The model has two time periods, where utility derived in the second period is discounted with the discount factor $\delta$, with $\delta \in(0,1)$. At the beginning of period 1, Nature draws a state of the world $s$, which is the same for both jurisdictions, for each jurisdiction the type of an incumbent government $I_{i} \in$ $\{G, B\}$, where $G$ stands for 'good' and $B$ for 'bad', and for each jurisdiction a variable $S_{i} \in\{L, H\}$ indicating whether the policy payoffs, given a state of the world, are low $\left(S_{i}=L\right)$ or high $\left(S_{i}=H\right)$. For example, suppose state 1 has been realized and $S_{i}=H$ has been drawn for jurisdiction $i$. The incumbent in $i$ then has to choose between the new policy with payoff $\pi+3 \Delta$ and the old policy with payoff $\pi+\Delta$. If, in contrast, $S_{i}=L$ is drawn, the choice is between $\pi+2 \Delta$ from the new policy and $\pi$ from the old policy.

Let $\theta$ be the probability that state 2 is realized, and $\operatorname{Pr}\left(I_{i}=G\right)=\gamma$ $(i=1,2)$ the probability that a good incumbent is drawn in jurisdiction $i$. The draws of $I_{1}$ and $I_{2}$ are independent. The variables $S_{1}$ and $S_{2}$, determining the policy payoffs conditional on state $s$ in both jurisdictions, are distributed according to the joint density

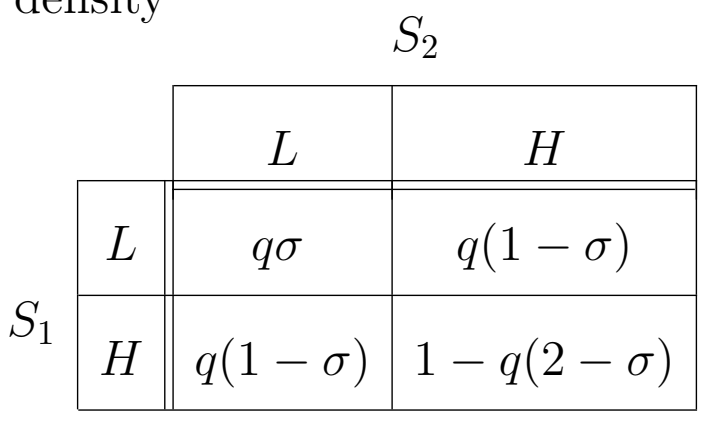

where $\sigma \equiv \operatorname{Pr}\left[S_{i}=L \mid S_{j}=L\right], i \neq j$. Thus $q$ is a jurisdiction's (unconditional) probability of having $S=L$. Since we do not want to deal with the case of negative correlation between $S_{1}$ and $S_{2}$, let $\sigma$ be restricted by $\sigma \in[q, 1]$. With $\sigma=1$ we have perfect correlation, whereas with $\sigma=q$ we have independence. ${ }^{4}$

After $s, I_{i}$ and $S_{i}(i=1,2)$ have been drawn, incumbents in both jurisdiction simultaneously choose a pair $\left(p_{i}, \tau_{i}\right)$. Policy payoffs are realized,

\footnotetext{
${ }^{4}$ This density has been used in a model contained in the working paper version of Bordignon, Cerniglia, and Revelli (2003).
} 
and the utility $v_{i}=\pi_{i}-\tau_{i}$ is delivered to the voters. At the end of period 1, elections take place. In each jurisdiction, the resident either reelects the incumbent or chooses a challenger who is drawn from the same distribution as incumbents. The voting is retrospective, and resident-voters care about maximizing their period-2 utility. In period 2, governments (either reelected incumbents or newly elected challengers) once again choose a policy and a transfer to themselves.

Information is distributed as follows. All underlying distributions are common knowledge. The draws of $s$ and $I_{i}, i=1,2$ are revealed to both jurisdiction's incumbents. Thus each incumbent knows the state of the world, its own type and the type of the other jurisdiction's incumbent. ${ }^{5}$ Furthermore, each incumbent observes the realization of $S_{i}$ in his own jurisdiction. Consequently, when simultaneously choosing $\left(p_{i}, \tau_{i}\right)$, incumbents in $i=1,2$ have perfect information about the payoff $\pi_{i}$ their policy $p_{i}$ will generate in their own jurisdiction. With regard to policy payoffs in the other jurisdiction, incumbents know which policy is superior and the distribution of payoffs conditional on the incumbent's policy choice in the neighboring jurisdiction. Residents in both jurisdictions do not know neither incumbents' types nor the realizations of $s$ and $S_{i}, i=1,2$. What the resident in $i$ observes is the policy in $i$ and his utility $v_{i}$, i.e. the payoff from $p_{i}$ net of the transfer taken by the incumbent. If (and only if) we allow for yardstick competition, residents can also observe the policy and the resident's utility in the other jurisdiction.

\footnotetext{
${ }^{5}$ The assumption that incumbents know each other's type is the one Besley and Case (1995) use. That incumbents can condition their strategies on the type of incumbent in the other jurisdiction is particularly important for the empirical implication of yardstick competition to generate spatial correlation in policy innovations.
} 


\section{Analysis of equilibria}

Two equilibria will be derived, one without comparative performance evaluation (voters cannot observe the policy choice and the resident's utility level in the other jurisdiction) and one with yardstick competition, where voters base their voting rule on the observation of incumbents' performance in both jurisdictions.

\subsection{An equilibrium without yardstick competition}

Without yardstick competition, there is no link between both jurisdictions. The Perfect Bayesian Equilibrium described in proposition 1 is thus an equilibrium in the behavior of incumbents and resident-voters if we look at each of both jurisdictions separately. Denote the strategies of incumbents ${ }^{6}$ by $\mu\left[s, S_{i}, I_{i}\right]=\left(p_{i}, \tau_{i}\right)$ and the strategies of residents as voters by $\varsigma\left(p_{i}, v_{i}\right) \in[0,1]$. With respect to out-of-equilibrium beliefs of voters the following simple rule is specified: Whenever it is apparent that a transfer has been taken and/or it is apparent that the inferior policy has been chosen in $i$, the voter in $i$ believes that $I_{i}=B .^{7}$ For notational convenience, define the sets $C_{i}=$ $\{(n, \pi+3 \Delta),(n, \pi+2 \Delta),(o, \pi+\Delta),(o, \pi)\}$ and $D_{i}=C_{i} \backslash\{(o, \pi)\}, i=1,2$. $C_{i}$ is the set of all pairs $\left(p_{i}, v_{i}\right)$ which, for some draw of $s$ and $S_{i}$, contain as elements the superior policy and the corresponding utility of the voter given a zero transfer. Note that the index for jurisdictions is suppressed in the remainder of this subsection.

Although good incumbents behave non-strategically, for completeness the description of their behavior is included in the following proposition.

\footnotetext{
${ }^{6}$ Strictly speaking this labelling is not correct, since second period choices of governments are not described. These are trivial, however, and the paper follows the literature in suppressing them from what is called incumbents' strategies.

${ }^{7}$ Under proposition 1 , pairs $(p, v)$ which do not occur on the equilibrium path will necessarily reveal that either a transfer has been taken or the inferior policy has been chosen, or both.
} 
Proposition 1 Define $\theta^{*} \equiv(1-q) / q, \delta^{*}=1-\Delta / \bar{\tau}$ and suppose $q \geq 1 / 2$, $\theta \geq \theta^{*}$ and $\delta \geq \delta^{*}$. Then there exists a Perfect Bayesian Equilibrium with the following strategies:

Good incumbents set

$$
\begin{aligned}
& \text { (i) } \mu(1, H, G)=\mu(1, L, G)=(n, 0), \\
& \text { (ii) } \mu(2, H, G)=\mu(2, L, G)=(o, 0),
\end{aligned}
$$

bad incumbents set

$$
\begin{aligned}
& \text { (iii) } \mu(1, H, B)=\mu(2, H, B)=(o, \Delta), \\
& \text { (iv) } \mu(1, L, B)=\mu(2, L, B)=(o, \bar{\tau}),
\end{aligned}
$$

and the representative voter sets

(v) $\varsigma[(p, v) \in C]=1$,

(vi) $\varsigma[(p, v) \notin C]=0$.

Proof. We begin by checking for profitable deviations of the voter. Since good incumbents maximize the voter's utility and bad incumbents according to the proposed strategy never play in a way such that $(p, v) \in D$, reelecting the incumbent after observing $(p, v) \in D$ and not reelecting after observing $(p, v) \notin C$ is optimal for the voter. Let $\operatorname{Pr}(I=G \mid p, v)$ denote the voter's beliefs. After observing $(o, \pi)$, the probability of having a good government in office is $\operatorname{Pr}(I=G \mid o, \pi)=\frac{\gamma \theta q}{\gamma \theta q+(1-\gamma)(1-q)}$. This is at least as high as $\gamma$, the probability of drawing a good challenger, if $\theta \geq \theta^{*}$. Since $q \geq 1 / 2$, it is guaranteed that $\theta^{*} \leq 1$.

Now we have to check for profitable deviations of bad incumbents. For a bad incumbent having $S=L$ playing $(o, \bar{\tau})$ dominates any other action: Reelection can only be gained by taking a zero transfer in the first period, which cannot be optimal given that the utility from taking the maximum transfer in the second period is $\delta \bar{\tau}$. Since choosing the new policy is costly, it 
can also not be optimal to play $p=n$ and take some transfer $\tau \in\{0, \Delta, \bar{\tau}\}$. If $S=H$, a bad government receives utility $\Delta+\delta \bar{\tau}$ by following the proposed strategy. As before, playing $p=n$ instead cannot be optimal since $e>0$. Finally, given that $\delta \geq \delta^{*}$, a deviation to $(o, \bar{\tau})$ is not profitable.

The intuition for this equilibrium is simple. Bad governments want to extract the highest possible transfer in the first period, but at the same time they seek reelection. With a sufficiently high discount factor, a mimicking behavior in the case of $S=H$ becomes worthwhile: The cost of a reduced transfer in the first period is outweighed by the benefit of winning reelection and being able to extract the maximum transfer in the second period. Since using the new policy is costly in terms of additional effort, in their mimicking behavior bad governments prefer the old policy even if the new one generates higher payoffs. For the voter, reelecting governments pretending that the old policy is superior and that payoffs are low is optimal as long as the probabilities that state 2 has been realized and that payoffs are low are sufficiently high.

\subsection{An equilibrium with yardstick competition}

For the case with yardstick competition, denote strategies of the incumbent in $i$ by $\mu\left(s, S_{i}, I_{i} ; I_{j}\right)=\left(p_{i}, \tau_{i}\right)$ and the strategies of the voter in $i$ by $\varsigma\left(p_{i}, v_{i} ; p_{j}, v_{j}\right) \in[0,1], i, j=1,2, i \neq j$. Since good incumbents behave as before, the description of their behavior is suppressed in the following proposition. Assume that out-of-equilibrium beliefs of voters are formed according to the following rules: (i) Whenever, for some jurisdiction $i$, it is apparent that a transfer has been taken and/or it is apparent that the inferior policy has been chosen, voters in both jurisdictions believe that $I_{i}=B$. (ii) If voters observe a combination $\left(p_{1}, v_{1}\right),\left(p_{2}, v_{2}\right)$ which does not occur on the equilibrium path, while complying to (i) they use Bayes' Rule whenever possible to form their beliefs with respect to the types 
of incumbents holding office. ${ }^{8}$ For notational convenience, define the sets $E_{i}=\left\{\left(p_{i}, v_{i}\right): p_{i}=n, v_{i} \in\{\pi+3 \Delta, \pi+2 \Delta, \pi+3 \Delta-\bar{\tau}, \pi+\Delta, \pi+2 \Delta-\bar{\tau}\}\right\}$, $i=1,2$. The crucial property of $E_{i}$ is that after observing some $\left(p_{i}, v_{i}\right) \in E_{i}$, voters in both jurisdictions know that the new policy is superior.

Proposition 2 Define $\delta^{* *}=1-\frac{\Delta-e}{\bar{\tau}}, \sigma^{* *}=\frac{3 q-1}{2 q}$ and $\gamma^{* *}=\frac{1-q(2-\sigma+\theta(1-\sigma))}{1-q(2-\sigma+\theta(2-3 \sigma))}$, and suppose $q>1 / 2, \theta \geq \theta^{*}, \sigma \leq \sigma^{* *}, \gamma \geq \gamma^{* *}$ and $\delta \geq \delta^{* *}$. Then there exists a Perfect Bayesian Equilibrium with the following strategies:

Bad incumbents set

(i) $\mu(1, H, B ; B)=(o, \Delta)$,

(ii) $\mu(1, H, B ; G)=(n, \Delta)$,

(iii) $\mu(1, L, B ; B)=\mu(1, L, B ; G)=\mu(2, L, B ; B)=\mu(2, L, B ; G)=(o, \bar{\tau})$,

(iv) $\mu(2, H, B ; B)=\mu(2, H, B ; G)=(o, \Delta)$,

and the representative voters sets

(v) $\varsigma\left[\left(p_{i}, v_{i}\right) \in\{(n, \pi+3 \Delta),(n, \pi+2 \Delta)\} ; \cdot\right]=1$,

(vi) $\varsigma\left[\left(p_{i}, v_{i}\right) \in\{(o, \pi+\Delta),(o, \pi)\} ;\left(p_{j}, v_{j}\right) \in E_{j}\right]=0$,

(vii) $\varsigma\left[\left(p_{i}, v_{i}\right) \in\{(o, \pi+\Delta),(o, \pi)\} ;\left(p_{j}, v_{j}\right) \notin E_{j}\right]=1$,

(viii) $\varsigma\left[\left(p_{i}, v_{i}\right) \notin C_{i} ; \cdot\right]=0$.

Proof. Once again we begin by checking for profitable deviations of the voter. Let $\operatorname{Pr}\left(I_{i}=G \mid p_{i}, v_{i} ; p_{j}, v_{j}\right)$ denote beliefs of voters. As before, if the voter observes $(n, \pi+3 \Delta)$ in his own jurisdiction, he believes a good incumbent to be in office with probability one. For $\left(p_{i}, v_{i}\right)=(n, \pi+2 \Delta)$, we have to consider three cases. Firstly, $\operatorname{Pr}\left(I_{i}=G \mid n, \pi+2 \Delta ; n, \pi+3 \Delta\right)=\frac{\gamma q(1-\sigma)}{\gamma q(1-\sigma)+(1-\gamma)(1-q(2-\sigma))}$. This is at least as high as $\gamma$ since $\sigma \leq \sigma^{* *}$. Furthermore, $\sigma^{* *} \geq q \forall q \in\left[\frac{1}{2}, 1\right]$,

\footnotetext{
${ }^{8}$ As we will see, for all possible combinations $\left(p_{1}, v_{1}\right),\left(p_{2}, v_{2}\right)$ off the equilibrium path under proposition 2 , beliefs with respect to the type of the incumbent in at least one jurisdiction are given by (i).
} 
such that for all such $q$ there exists some $\sigma \in\left[\frac{1}{2}, 1\right]$ for which $q \leq \sigma \leq \sigma^{* *}$ holds. Secondly, $\operatorname{Pr}\left(I_{i}=G \mid n, \pi+2 \Delta ; n, \pi+2 \Delta\right)=\frac{\gamma \sigma+(1-\gamma)(1-\sigma)}{\gamma \sigma+2(1-\gamma)(1-\sigma)}$, which is at least as high as $\gamma$ given that $\sigma \geq 1 / 2$. Thirdly, after observing $(n, \pi+$ $\left.2 \Delta ;\left(p_{j}, v_{j}\right) \notin\{(n, \pi+3 \Delta),(n, \pi+2 \Delta)\}\right)$, reelecting the incumbent is optimal since by applying Bayes' Rule and - if necessary - the out-of-equilibrium beliefs, in all cases we find that the probability of having a good incumbent in office is one.

Part (vi) of the voter's strategy reflects out-of-equilibrium beliefs. Since the observation of each of the elements in $E_{j}$ reveals that the new policy is superior, it is optimal not to reelect a government in $i \neq j$ choosing $p_{i}=o$.

With regard to part (vii) of the voters strategy, first note that bad governments according to the proposed strategies never play $(o, \pi+\Delta)$. As long as $\left(p_{j}, v_{j}\right) \notin E_{j}$, it is therefore optimal to reelect the incumbent in $i \neq j$ after observing $\left(p_{i}, v_{i}\right)=(o, \pi+\Delta)$. Furthermore, note that $\operatorname{Pr}\left(I_{i}=\right.$ $G \mid o, \pi ; o, \pi+\Delta)=\operatorname{Pr}\left(I_{i}=G \mid n, \pi+2 \Delta ; n, \pi+3 \Delta\right)$. Thus it is optimal to reelect the incumbent in $i$ after observing $(o, \pi ; o, \pi+\Delta)$ given that $\sigma \leq \sigma^{* *}$. If voters observe $(o, \pi)$ in both jurisdictions, the probability of having a good government is $\frac{\gamma^{2} \theta q \sigma+\gamma(1-\gamma) \theta q(1-\sigma)}{\gamma^{2} \theta q \sigma+2 \gamma(1-\gamma) \theta q(1-\sigma)+(1-\gamma)^{2}(1-q(2-\sigma))}$. This is at least as high as $\gamma$ given that $\gamma \geq \gamma^{* *}$. Note that the denominator in $\gamma^{* *}$ is positive for all $\theta \in\left[\theta^{*}, 1\right]$ given that $q \in\left(\frac{1}{2}, 1\right]$ and $\sigma \in\left[q, \sigma^{* *}\right]$, and that $\gamma^{* *} \leq 1 \forall \sigma \geq 1 / 2$. If $(o, \pi ; o, \pi-\bar{\tau})$ is observed, the probability for the voter in $i$ of having a good government in office is $\frac{\gamma \theta \sigma}{\gamma \theta \sigma+(1-\gamma)(1-\sigma)}$. This is at least as high as $\gamma$ for all $\theta \in\left[\theta^{*}, 1\right]$ given that $\sigma \geq q$. Finally, applying Bayes' Rule together with the out-of-equilibrium beliefs specified above shows that for all remaining $\left(p_{j}, v_{j}\right) \notin E_{j}$ it is optimal to reelect the incumbent after observing $(o, \pi)$ in $i$.

Part (viii) of the voters strategy is optimal since by observing $\left(p_{i}, v_{i}\right) \notin C_{i}$ it is revealed that either a transfer has been taken or the inferior policy has been chosen in $i$, or both.

Now we check for profitable deviations of bad incumbents. It is easy to see that, if both governments are bad, policy choices and transfers identical 
to those described in proposition 1 together with the specified beliefs and the proposed voting rule constitute an equilibrium in the presence of yardstick competition. If the other government is good, however, it is not longer optimal for a bad incumbent in $i$ to choose $(o, \Delta)$ if $s=1$ and $S_{i}=H$ since the superior new policy will be used in $j$, leading the voter in $i$ to vote for the challenger. Instead, it is now optimal for $i$ 's incumbent to set $(n, \Delta)$, to deliver $v_{i}=\pi+2 \Delta$ to the voter and gain reelection: Since $\delta \geq \delta^{* *}$, the utility $\Delta-e+\delta \bar{\tau}$ is at least as high as the one from the best alternative, $(o, \bar{\tau})$. If $s=2$ and $S_{i}=H,(o, \Delta)$ remains the optimal choice since given the proposed voting rule the probability of reelection is one. If $S_{i}=L$ and the other government is good, the same reasoning as in the proof of proposition 1 applies.

The intuition for the yardstick equilibrium is straightforward. By now being able to compare policies in two jurisdictions, voters have additional information to base their reelection decision on. In state 1, a bad incumbent knowing that the government in the other jurisdiction is good is now forced to choose the new policy to gain reelection. Since the performance of the good government will reveal that the new policy is superior, using the old policy would result in the government being removed from office. The voter thus benefits from the additional information available by getting the superior policy more often. The selection properties, i.e. the probabilities of finding out a bad incumbent, are the same in both equilibria.

The condition on the discount factor $\delta$ is of course stronger for the yardstick equilibrium than for the equilibrium without comparative performance evaluation. The reason is that, if the other government is good and the new policy must be used for a successful mimicking, the additional effort $e$ has to be invested to perform the public policy. Note, however, that the role of $e$ is only to make the new policy, other things being equal, less attractive for bad governments than the old policy. Thus $e$ might be arbitrarily small, and $\lim _{e \rightarrow 0} \delta^{* *}=\delta^{*}$. If the new policy is superior, payoffs are high and 
the other government is bad, or if the old policy is superior and payoffs are high, bad governments imitate good governments in equilibrium by choosing $(o, \Delta)$ and delivering $v=\pi$. Consequently, a sufficiently high $\gamma$ is necessary to make reelection the optimal choice for the voter after observing $(o, \pi)$ in both jurisdictions.

The most interesting parameter is $\sigma$. The yardstick equilibrium is supported by values of $\sigma \in\left[q, \sigma^{* *}\right]$, i.e. the positive correlation between $S_{1}$ and $S_{2}$ must be sufficiently low. The intuition is that it must be worthwhile for the voter in $i$ to reelect an incumbent using the same policy as the neighboring jurisdiction's incumbent but delivering a utility equal to the low payoff compared to a utility equal to the high payoff delivered to the voter in $j$. As a counterexample, consider the case with perfect correlation $[\sigma=1]$ between $S_{1}$ and $S_{2}$ for some $q \in\left(\frac{1}{2}, 1\right)$. In this situation, it cannot be optimal for the voter in $i$ to reelect an incumbent choosing $p_{i}=n$ and delivering, say, $v_{i}=\pi+2 \Delta$ given that $v_{j}=\pi+3 \Delta$ since it is apparent that a transfer has been taken in $i$.

In general, benevolent governments in other jurisdictions introduce a particularly rigorous yardstick for government performance limiting the level of rents that can be extracted by office-motivated governments seeking a second term of office. Consider a situation in which, as in the model above, the rents governments can earn are the same in both periods. Suppose furthermore that the level of rents which can be earned in the first period without forgoing reelection, given that the government in the other jurisdiction is good, is reduced to zero. ${ }^{9}$ Rent-seeking governments will then take the maximum rent they can get in the first period and forgo reelection. Under these circumstances, yardstick competition forces bad governments to behave, from the point of view of voters, worse with respect to first-period performance than

${ }^{9}$ An example would be the model with $\sigma=1$. 
in a situation without comparative performance evaluation. ${ }^{10}$ The model from section 2 illustrates an effect of yardstick competition which is more in line with the original Brennan and Buchanan (1977) view: Forcing bad governments to use more caution when they engage in rent seeking. In the equilibrium described above, bad governments manage to extract rents without forgoing reelection even in cases where the yardstick is set by a good government. But in some cases they now have to choose the superior new policy in order to gain reelection. Yardstick competition in this model thus serves as a discipline device for rent-seeking governments. In our example, the benefit to voters comes as a higher probability of having a government choosing optimal policies.

The yardstick competition equilibrium has an interesting empirical implication. It seems plausible that in any given jurisdiction, voters are better informed about governments' performance in nearby jurisdictions than in more distant jurisdictions. Hence, we should expect yardstick competition between jurisdictions to make local decisions in the adoption of policy innovations depending on expected policies is neighboring jurisdictions. Empirically, this would mean positive spatial correlation in policy innovations across jurisdictions. The simplest way to test this prediction would be to define innovative versus traditional policies in a particular policy field and to check whether the distribution of policy innovations across jurisdictions shows positive spatial correlation or not. In practice, however, things may not be that easy, because most local governments are active in various policy fields and fulfill lots of different tasks. Hence, governments may choose a mix between innovative policies for some tasks and more traditional approaches for others. Furthermore, the importance of various fields in public policy may vary across jurisdictions. For instance, some tasks may be common to all jurisdictions, while others may be specific for governments in certain regions.

\footnotetext{
${ }^{10}$ In such a model, voters gain from yardstick competition by an increased probability of finding out bad governments.
} 
This, of course, will feed back to jurisdictions and their choice of policies. Hence, a more general model will be useful in order to come up with testable hypotheses on the strategic behavior of jurisdictions in the adoption of policy innovations.

\section{Yardstick competition with common and jurisdiction-specific policies}

In the following, a straightforward extension of the theoretical analysis will be presented. It covers asymmetric jurisdictions and a multi-dimensional policy space. The empirical implications derived from this theory will also be briefly discussed.

Consider the model from section 2 with the extension that we now have two tasks $k=A, B$. Suppose that $A$ is a common task which has to be fulfilled in both jurisdictions and let $B$ be a task which is specific for jurisdiction 2 and which is not a policy task in jurisdiction 1. Clearly, with regard to the specific task, yardstick competition between jurisdictions is impossible. However, yardstick competition may takes place with respect to the common task.

The government in $i=1$ has to make the same choice as before. In $i=2$ the government now has to choose two policies $p_{2 A}$ and $p_{2 B}$. For each task, the choice is between an old and a new policy. Assume that task-specific state variables $s_{k} \in\{1,2\}, k=A, B$ are independently drawn from the same distribution. $\theta$ is now the probability that $s_{k}=2$ is drawn for task $k=A, B$. If $s_{k}=1\left[s_{k}=2\right]$, the new [old] policy is superior for task $k$. As in the model with a one-dimensional policy space, policy payoffs are either low or high depending on the draw for $S_{i} \in\{L, H\}$. Together with the policies $p_{2 A}$ and $p_{2 B}$, task-specific transfers $\tau_{2 A}, \tau_{2 B} \in\{0, \Delta, \bar{\tau}\}$ must be chosen. The per-period utility of the government in $i=2$ from performance in task $k$ now 
is

$$
u_{2 k}\left(p_{2 k}, \tau_{2 k}\right)= \begin{cases}\tau_{2 k}-e & \text { if } p_{2 k}=n \\ \tau_{2 k} & \text { if } p_{2 k}=o\end{cases}
$$

In the situation without yardstick competition little is changed compared to subsection 3.1. In $i=1$, proposition 1 holds. For $i=2$, it is easy to show that an equilibrium exists where

(i) bad governments choose $(o, \Delta)$ for both tasks if $S_{1}=H$;

(ii) bad governments choose $(o, \bar{\tau})$ for both tasks if $S_{1}=L$;

(iii) the voter opts for the challenger if it is apparent that a transfer has been taken or it is apparent that the inferior policy has been chosen for some task $k=A, B$, or both, and reelects the incumbent otherwise.

For the situation with yardstick competition, let the strategies of incumbents in $i=1$ be denoted by $\mu\left(s_{A}, S_{i}, I_{i}\right)=\left(p_{1 A}, \tau_{1 A}\right)$, while strategies of incumbents in $i=2$ are denoted as $\mu\left(s_{A}, s_{B}, S_{i}, I_{i}\right)=\left(\mu_{A}, \mu_{B}\right)$, where $\mu_{k}=\left(p_{2 k}, \tau_{2 k}\right), k=A, B$. Assume out-of-equilibrium beliefs defined by analogy to the model with yardstick competition in a single common policy.

Proposition 3 Define $\tilde{\sigma}=1 /\left(1+\theta^{2}\right), \tilde{\gamma}=\frac{1-q\left(2-\sigma+\theta^{2}(1-\sigma)\right)}{1-q\left[2-\sigma+\theta^{2}(1-\sigma)+\theta(1-\sigma(1+\theta))\right]}, \tilde{\theta}=$ $[1-q(2-\sigma)] /[q(1-\sigma)]$ and $\tilde{\tilde{\theta}}=[(1-q) /(3 q-1)]^{\frac{1}{2}}$ and suppose

(a) $q \geq 1 / 2$,

(b) $\theta \geq \max \{\tilde{\theta}, \tilde{\tilde{\theta}}\}$

(c) $\tilde{\sigma} \leq \sigma \leq \sigma^{* *}$,

(d) $\delta \geq \delta^{* *}$,

(e) $\gamma \geq \tilde{\gamma} \quad$ if $1-q\left[2-\sigma+\theta^{2}(1-\sigma)+\theta(1-\sigma(1+\theta))\right]>0$.

Then there exists a Perfect Bayesian Equilibrium with the following strategies:

Bad incumbents and voters in $i=1$ follow their strategies described in proposition 2. 
(i) $\quad \mu_{k}(1, H, B ; B)=(o, \Delta), \quad k=A, B$

(ii) $\mu_{A}(1, H, B ; G)=(n, \Delta)$,

$$
\mu_{B}(1, H, B ; G)=(o, \Delta),
$$

(iii) $\quad \mu_{k}(2, H, B ; B)=\mu_{k}(2, H, B ; G)=(o, \Delta), \quad k=A, B$

(iv) $\mu_{k}(1, L, B ; B)=\mu_{k}(1, L, B ; G)=$

$$
\mu_{k}(2, L, B ; B)=\mu_{k}(2, L, B ; G)=(o, \bar{\tau}), \quad k=A, B .
$$

Voters in $i=2$ vote for the challenger whenever for some task $k=A, B$ it is apparent that a transfer has been taken or if it is apparent that the inferior policy has been chosen in $i$, or both. In all other cases, the incumbent is reelected.

Proof. See the appendix.

From proposition 3 we see that the role of yardstick competition as a discipline device for rent-seeking politicians carries over from the simple to a richer model with various political tasks of governments and varying degrees of yardstick competition across tasks. However, in a model with only a subset of tasks being subject to yardstick competition the pattern of government performance becomes richer. Even benevolent governments now switch from innovative to conservative policies across tasks. Moreover, rentseeking governments can stick to conservative policies and avoid innovations for jurisdiction-specific tasks where comparative performance evaluation by voters is hampered. This gives rent-seeking governments additional possibilities to masquerade as acting in the interest of the voter. Of course, for common tasks the same discipline-enhancing effect of comparative performance evaluations by voters is at work: if a new policy is shown to be superior by successfully innovating good governments in the neighborhood, bad governments seeking reelection are forced to use the innovation, too. 
The empirical implications of the extended model are the following: Firstly, the share of tasks where innovative policies are used should on average be higher with yardstick competition than without. Hence, governments should implement innovative policies more often if policy competition is stronger. Secondly, the share of tasks where governments use innovative policies should show spatial correlation. Finally, the model suggests that the degree of spatial correlation in policy innovations varies across lpolicies with the intensity of policy competition.

\section{Conclusion}

This paper offers a simple theoretical framework for the analysis of strategic interaction between jurisdictions with respect to policy innovations. Furthermore, the paper provides testable hypotheses on spatial patterns in the adoption of policy innovations by jurisdictions in a situation where information on the prospects of new vs. old policies is asymmetrically distributed between rent-seeking governments and voters.

For the simple model with yardstick competition between jurisdictions, the existence of a Perfect Bayesian Equilibrium has been shown. In this equilibrium, rent-seeking governments are disciplined by voters who are able to compare the performance of politicians across jurisdictions. With yardstick competition, the optimal policy is chosen more often compared to the equilibrium without yardstick competition, while the level of rents extracted by incumbents as well as the selection properties are the same in both equilibria. Hence, the voters in this model are better off with yardstick competition than without.

The basic model has also been extended to cover a situation where governments have to fulfill multiple political tasks with varying degrees of yardstick competition across tasks. We have seen that the role of yardstick competition as a discipline device for rent-seeking politicians carries over from the 
basic to the more involved model with only a subset of tasks being subject to yardstick competition. However, the pattern of government performance becomes richer, with governments now switching from innovative to conservative policies across tasks in equilibrium. In the yardstick competition equilibria, the force driving rent-seeking governments to perform the innovative policy (given that it is optimal to do so) is the following: knowing that suc-

cessfully innovating benevolent governments in the neighborhood will reveal the new policy being superior, rent-seeking governments are forced to use the innovation in order to build up a reputation sufficiently strong to gain reelection. The paper has thus presented a theoretical example where competition between independent governments improves the political system's properties with regard to the selection of optimal policies in a discrete choice problem.

In future empirical work on the behavior of jurisdictions in the adoption of policy innovations, the hypotheses provided by the yardstick competition model should be tested. An empirical literature on the question how jurisdictions influence each other in the choice between 'traditional' and 'new' policies is largely missing. This is somewhat surprising, given that Walker (1969) has addressed the question already many years ago and that both researchers and practitioners should be interested in learning more on competition between jurisdictions with respect to the adoption of political best-practice technologies.

\section{References}

Belleflamme, P., and J. Hindriks (2002): "Yardstick Competition and Political Agency Problems," CORE Discussion Paper 2002/29, Universite Catholique de Louvain.

Besley, T., and A. Case (1995): "Incumbent Behavior: Vote-Seeking, Tax-Setting, and Yardstick Competition," The Amercian Economic Review, 85(1), 25-45. 
Besley, T., and M. Smart (2002): "Does Tax Competition Raise Voter Welfare?," CEPR Discussion Papers 3131.

Bordignon, M., F. Cerniglia, and F. Revelli (2003): "In Search of Yardstick Competition: A Spatial Analysis of Italian Municipality Property Tax Setting," Journal of Urban Economics, 54, 199-217.

Brennan, G., and J. M. Buchanan (1977): "Towards a Tax Constitution for Leviathan," Journal of Public Economics, 8, 255-273.

Conte, S., and S. Morris (1995): "On the Form of Transfers to Special Interests," Journal of Political Economy, 105, 1210-1235.

Holmstrom, B. R. (1982): "Moral Hazard in Teams," Bell Journal of Economics, 13, 324-40.

Kotsogiannis, C., and R. Schwager (2003): "Political Uncertainty and Policy Innovation," Mimeo, University of Exeter.

(2004): "Policy Innovation in Federal Systems," Mimeo, University of Exeter.

Walker, J. L. (1969): "The Diffusion of Innovations Among the American States," The American Political Science Review, 63(3), 880-899.

Wrede, M. (2001): "Yardstick Competition to Tame the Leviathan," European Journal of Political Economy, 17, 705-721. 


\section{Appendix}

\section{Proof of proposition 3:}

We will be brief since the proof is similar to the one for proposition 2 . We start with the voter in $i=2$. Let $\operatorname{Pr}\left[I_{2}=G \mid\left(p_{2 A}, v_{2 A}\right),\left(p_{2 B}, v_{2 B}\right) ;\left(p_{1 A}, v_{1 A}\right)\right]$ denote his beliefs. Consider the following five cases on the equilibrium path:

(I) $\operatorname{Pr}\left[I_{2}=G \mid(n, \pi+2 \Delta),(o, \pi) ;(n, \pi+3 \Delta)\right]=\frac{\gamma \theta q(1-\sigma)}{\gamma \theta q(1-\sigma)+(1-\gamma)(1-q(2-\sigma))}$. This is at least as high as $\gamma$ given that $\theta \geq \tilde{\theta}$. Since $\sigma \leq \sigma^{* *}$ we have $\tilde{\theta} \leq 1$.

(II) $\operatorname{Pr}\left[I_{2}=G \mid(n, \pi+2 \Delta),(o, \pi) ;(n, \pi+2 \Delta)\right]=\frac{\gamma \theta \sigma+(1-\gamma) \theta(1-\sigma)}{\gamma \theta \sigma+(1-\gamma)(1-\theta)(1-\sigma)}$. This is at least as high as $\gamma$ given that $\theta \geq \frac{\gamma(1-\sigma)}{\gamma \sigma+(1-\gamma)(1-\sigma)}$, which is implied by $\theta \geq \tilde{\theta}$.

(III) $\operatorname{Pr}\left[I_{2}=G \mid(o, \pi),(o, \pi) ;(o, \pi+\Delta)\right]=\frac{\gamma \theta q(1-\sigma)}{\gamma \theta q(1-\sigma)+(1-\gamma)(1-q(2-\sigma))}$. See (I).

(IV) $\operatorname{Pr}\left[I_{2}=G \mid(o, \pi),(o, \pi) ;(o, \pi)\right]=\frac{\gamma^{2} \theta^{2} q \sigma+\gamma(1-\gamma) \theta^{2} q(1-\sigma)}{\gamma^{2} \theta^{2} q \sigma+\gamma(1-\gamma) \theta(1+\theta) q(1-\sigma)+(1-\gamma)^{2}(1-q(2-\sigma))}$.

This is at least as high as $\gamma$ if the denominator in $\tilde{\gamma}$ is smaller than or equal to zero given that $\sigma \geq 1 /(1+\theta)$, which is implied by $\sigma \geq \tilde{\sigma}$. $\theta \geq \tilde{\tilde{\theta}}$ guarantees that for all $q \in[0.5,1]$ there exists some $\sigma$ such that $1 /(1+$ $\theta) \leq \sigma \leq \sigma^{* *}$ holds. If the denominator in $\tilde{\gamma}$ is strictly positive we have $\operatorname{Pr}\left[I_{2}=G \mid(o, \pi),(o, \pi) ;(o, \pi)\right] \geq \gamma$ since $\gamma \geq \tilde{\gamma}$. With $\sigma \geq 1 /(1+\theta)$ it is guaranteed that $\tilde{\gamma} \leq 1$.

(V) $\operatorname{Pr}\left[I_{2}=G \mid(o, \pi),(o, \pi) ;(o, \pi-\bar{\tau})\right]=\frac{\gamma \theta^{2} \sigma}{\gamma \theta^{2} \sigma+(1-\gamma)(1-\sigma)}$. This is at least as high as $\gamma$ given that $\sigma \geq \tilde{\sigma}$. $\theta \geq \tilde{\tilde{\theta}}$ guarantees that for all $q \in[0.5,1]$ there exists some $\sigma \in[0.5,1]$ such that $\tilde{\sigma} \leq \sigma \leq \sigma^{* *}$ holds.

In all remaining cases the optimality of the voting rule is immediately obvious from Bayes' Rule or given by the out-of-equilibrium beliefs. By repeating the same procedure for the voter in $i=1$ it is easy to show that the proposed voting rule is optimal given that the conditions specified above hold. The details are omitted.

For the incumbent in $i=1$ the situation is basically unchanged compared to the situation with an identical neighborhood jurisdiction. He must be willing to invest $e$ in order to gain reelection. Since $\delta \geq \delta^{* *}$ this is the case. The incumbent in $i=2$ must prefer the new policy for task $A$ if $s_{A}=1, S_{2}=H$ and $I_{1}=G$. This requires $\delta \geq 1-\left(\Delta-\frac{1}{2} e\right) / \tilde{\tau}$, which is implied by $\delta \geq \delta^{* *}$. 